\title{
Supermodularity in Unweighted Graph Optimization II: Matroidal Term Rank Augmentation
}

\author{
Kristóf Bérczi* and András Frank ${ }^{\dagger}$
}

March 31, 2018

\begin{abstract}
Ryser's max term rank formula with graph theoretic terminology is equivalent to a characterization of degree sequences of simple bipartite graphs with matching number at least $\ell$. In a previous paper [1] by the authors, a generalization was developed for the case when the degrees are constrained by upper and lower bounds. Here two other extensions of Ryser's theorem are discussed. The first one is a matroidal model, while the second one settles the augmentation version. In fact, the two directions shall be integrated into one single framework.
\end{abstract}

\section{Introduction}

Ryser [16] derived a formula for the maximum term rank of a $(0,1)$-matrix with specified row- and column-sums. In graph theoretic terms, his theorem is equivalent to a characterization for the existence of a degree-specified simple bipartite graph (bigraph for short) with matching number at least $\ell$. Several natural extensions, like the min-cost and the subgraph version, turned out to be NP-hard, but in a previous paper [1], we could extend Ryser's theorem to the degree-constrained case when, instead of exact degreespecifications, lower and upper bounds are imposed on the degrees of the bigraph. An even more general problem was also solved when, in addition, lower and upper bounds were imposed on the number of edges. The main tool in [1] for proving these extensions was a general framework for covering an intersecting supermodular function by degree-constrained simple bipartite graphs.

In the present paper we consider two other extensions of Ryser's theorem: the augmentation and the matroidal version. In the first one, a given initial bigraph is to be augmented to get a simple degreespecified bigraph with matching number at least $\ell$. In original matrix terms, this means that some of the entries of the $(0,1)$-matrix are specified to be 1 . The solvability of this version is in sharp contrast with the NP-completeness of another variation when some entries of the matrix are specified to be 0 . (This follows from the NP-completeness of the problem that seeks to decide whether an initial bigraph $G_{0}$ has a perfectly matchable degree-specified subgraph, see [11], [13], [14].)

In the matroidal extension of Ryser's theorem, there is a matroid on $\mathrm{S}$ and there is a matroid on $\mathrm{T}$, and the goal is to find a degree-specified simple bigraph including a matching that covers bases in both matroids. These results will be consequences of a general framework including both the augmentation and the matroidal cases.

The starting point in deriving the main result is the supermodular arc-covering theorem by Frank and Jordán [9] (Theorem 1 below). Since [9] describes a polynomial algorithm, relying on the ellipsoid method, to compute the optima in question, our matroidal term rank augmentation problem also admits a polynomial algorithm. One of the most important applications in [9] is the directed node-connectivity augmentation problem. Végh and Benczúr [17] developed for this special case a pretty intricate but purely combinatorial algorithm (not relying on the ellipsoid method). Although not mentioned explicitly in [17], their algorithm can probably be extended to work on the supermodular arc covering theorem when the function in question is $S T$-crossing supermodular, but the details have not been worked out. (In the special case of node-connectivity augmentation, this general oracle was realized via network flow computations.)

${ }^{*}$ MTA-ELTE Egerváry Research Group, Department of Operations Research, Eötvös University, Pázmány P. s. 1/c, Budapest, Hungary, H-1117. e-mail: berkri@cs.elte.hu .

${ }^{\dagger}$ MTA-ELTE Egerváry Research Group, Department of Operations Research, Eötvös University, Pázmány P. s. 1/c, Budapest, Hungary, H-1117. e-mail: frank@cs.elte.hu . 
Therefore the algorithm of Végh and Benczúr seems to be adaptable to the term rank problem, too. In a forthcoming paper [3], we shall develop a much simpler algorithm along with a natural unification of the matroidal augmentation and the degree-constrained term rank problems.

\subsection{Notions and notation}

We use the notation of [1]. Here we briefly repeat the most important notions. For a family $\mathcal{T}$ of sets, let $\cup \mathcal{T}$ denote the union of the members of $\mathcal{T}$. For a subpartition $\mathcal{T}=\left\{T_{1}, \ldots, T_{q}\right\}$, we always assume that its members $T_{i}$ are non-empty but $\mathcal{T}$ is allowed to be empty (that is, $q=0$ ).

An arc st enters or covers a set $X$ if $s \notin X, t \in X$. A digraph covers $X$ if it contains an arc covering $X$. Let $S$ and $T$ be two non-empty subsets of a ground-set $V$. By an $S T$-arc we mean an arc st with $s \in S$ and $t \in T$. Two sets $X$ and $Y$ are $S T$-independent if $X \cap Y \cap T=\emptyset$ or $S-(X \cup Y)=\emptyset$, that is, no $S T$-arc enters both sets. Two subsets $X$ and $Y$ are comparable if $X \subseteq Y$ or $Y \subseteq X$. Two non-comparable sets $X$ and $Y$ are $T$-intersecting if $X \cap Y \cap T \neq \emptyset$ and $S T$-crossing if $X \cap Y \cap T \neq \emptyset$ and $S-(X \cup Y) \neq \emptyset$. A set-function $p$ is called positively $T$-intersecting ( $S T$-crossing) supermodular if the supermodular inequality

$$
p(X)+p(Y) \leq p(X \cap Y)+p(X \cup Y)
$$

holds for $T$-intersecting (resp. $S T$-crossing) subsets $X$ and $Y$ for which $p(X)>0$ and $p(Y)>0$. The function is fully supermodular if the supermodular inequality holds for every pair $X$ and $Y$ of subsets.

For a function $m: V \rightarrow \mathbf{R}$, the set-function $\widetilde{m}$ is defined by $\widetilde{m}(X)=\sum[m(v): v \in X]$. A set-function $p$ can analogously be extended to families $\mathcal{F}$ of sets by $\widetilde{p}(\mathcal{F})=\sum[p(X): X \in \mathcal{F}]$.

The following min-max theorem of Frank and Jordán [9] will be a basic tool in the proof of the main theorem.

Theorem 1 (Supermodular arc-covering, set-function version). A positively ST-crossing supermodular set-function $p$ for which $p\left(V^{\prime}\right) \leq 0$ holds when no ST-arc enters $V^{\prime}$ can be covered by $\gamma$ (possibly parallel) $S T$-arcs if and only if $\widetilde{p}(\mathcal{I}) \leq \gamma$ holds for every $S T$-independent family $\mathcal{I}$ of subsets of $V$. There is an algorithm, which is polynomial in $|S|+|T|$ and in the maximum value of $p(X)$, to compute the minimum number of $S T$-arcs to cover $p$ and an $S T$-independent family $\mathcal{I}$ of subsets maximizing $\widetilde{p}(\mathcal{I})$.

Henceforth we assume that $S$ and $T$ are two disjoint non-empty sets and $V:=S \cup T$. Let $G^{*}=\left(S, T ; E^{*}\right)$ denote the complete bipartite graph on bipartition $(S, T)$. Let $D^{*}=\left(S, T ; A^{*}\right)$ be the digraph arising from $G^{*}$ by orienting each of its edges from $S$ to $T$, that is, $A^{*}$ consists of all $S T$-arcs. More generally, for a bigraph $H=(S, T ; F)$, let $\vec{H}=(S, T ; \vec{F})$ denote the digraph arising from $H$ by orienting each of its edges from $S$ toward $T$.

Throughout we are given a simple bigraph $H_{0}=\left(S, T ; F_{0}\right)$ serving as an initial bigraph to be augmented. For $E_{0}:=E^{*}-F_{0}$, the bigraph $G_{0}=\left(S, T ; E_{0}\right)$ is called the bipartite complement of $H_{0}$, that is, $F_{0}$ and $E_{0}$ partition $E^{*}$. Note that a bigraph $G=(S, T ; E)$ is a subgraph of $G_{0}$ precisely if the augmented bigraph $G^{+}=\left(S, T ; F_{0}+E\right)$ is simple. For $X \subseteq S$ and $Y \subseteq T$, let $d_{G_{0}}(X, Y)$ denote the number of edges of $G_{0}$ connecting $X$ and $Y$.

\section{Matroidal covering and augmentation}

Let $p_{T}$ be a positively intersecting supermodular set-function on $T$. In [1], we studied the problem of finding a simple degree-specified bigraph $G=(S, T ; E)$ covering $p_{T}$ in the sense that

$$
\left|\Gamma_{G}(Y)\right| \geq p_{T}(Y) \text { for every subset } Y \subseteq T
$$

where $\Gamma_{G}(Y)$ denotes the set of neighbours of $Y$. Here we consider a framework which is more general in two directions. First, for a given initial simple bigraph $H_{0}=\left(S, T ; F_{0}\right)$, we want to find a degree-specified bigraph $G$ in such a way that $G^{+}:=G+H_{0}$ is simple and covers $p_{T}$. This kind of problems is often referred to as augmentation problems to be distinguished from the synthesis problems where $F_{0}$ is empty. If $p_{T} \equiv 0$, the augmentation problem is equivalent to finding a degree-specified subgraph of the bipartite complement of $H_{0}$. 
Second, we extend the notion of covering to matroidal covering in the following sense. Let $M_{S}=\left(S, r_{S}\right)$ be a matroid on $S$ with rank function $r_{S}$. A bigraph $G$ is said to $M_{S}$-cover $p_{T}$ if

$$
r_{S}\left(\Gamma_{G}(Y)\right) \geq p_{T}(Y) \text { for every subset } Y \subseteq T \text {. }
$$

Clearly, when $M_{S}$ is the free matroid, we are back at the original notion of covering by a bigraph.

\section{$2.1 \quad$ Degree-specified matroidal augmentation}

Let $m_{V}=\left(m_{S}, m_{T}\right)$ be a degree-specification. A bigraph $G=(S, T ; E)$ is said to fit $m_{V}$ if $d_{G}(v)=$ $m_{V}(v)$ for every $v \in S \cup T$. Our main goal is to describe a characterization for the existence of a bigraph $G$ fitting $m_{V}$ so that $G+H_{0}$ is simple and $M_{S}$-covers $p_{T}$. The more general problem, when there are upper and lower bounds on $V$, will be discussed in [3]. This degree-constrained version was solved in [1] in the special case when $H_{0}$ has no edges and $M_{S}$ is the $\ell$-uniform matroid on $S$.

Our main result is as follows.

Theorem 2. We are given a simple bigraph $H_{0}=\left(S, T ; F_{0}\right)$, a matroid $M_{S}=\left(S, r_{S}\right)$, a positively intersecting supermodular set-function $p_{T}$ on $T$, and a degree-specification $m_{V}=\left(m_{S}, m_{T}\right)$ on $V:=S \cup T$ for which $\widetilde{m}_{S}(S)=\widetilde{m}_{T}(T)=\gamma$. There is a bigraph $G=(S, T ; E)$ fitting $m_{V}$ for which $G^{+}=G+H_{0}$ is simple and $M_{S}$-covers $p_{T}$ if and only if

$$
\begin{gathered}
\tilde{m}_{S}(X)+\tilde{m}_{T}(Y)-d_{G_{0}}(X, Y)+\sum_{i=1}^{q}\left[p_{T}\left(T_{i}\right)-r_{S}\left(X \cup \Gamma_{H_{0}}\left(T_{i}\right)\right)\right] \leq \gamma \\
\text { whenever } Y \subseteq T, X \subseteq S \text {, and } \mathcal{T}=\left\{T_{1}, \ldots, T_{q}\right\} \text { is a subpartition of } T-Y,
\end{gathered}
$$

where $G_{0}$ is the bipartite complement of $H_{0}$.

Proof. Proof. Necessity. Suppose that there is a requested bigraph $G=(S, T ; E)$ and let $G^{+}=(S, T ; E \cup$ $\left.F_{0}\right)$. Note that the simplicity of $G^{+}$is equivalent to the requirement that $G$ is a subgraph of $G_{0}$. Let $X \subseteq S$ and $Y \subseteq T$ be subsets and let $\left\{T_{1}, \ldots, T_{q}\right\}$ be a subpartition of $T-Y$. Let $W_{i}:=\Gamma_{G}\left(T_{i}\right)-\left[X \cup \Gamma_{H_{0}}\left(T_{i}\right)\right]=$ $\Gamma_{G^{+}}\left(T_{i}\right)-\left[X \cup \Gamma_{H_{0}}\left(T_{i}\right)\right]$. Then we have

$$
\begin{gathered}
p_{T}\left(T_{i}\right) \leq r_{S}\left(\Gamma_{G^{+}}\left(T_{i}\right)\right) \leq r_{S}\left(\Gamma_{H_{0}}\left(T_{i}\right) \cup X\right)+r_{S}\left(W_{i}\right) \leq \\
r_{S}\left(\Gamma_{H_{0}}\left(T_{i}\right) \cup X\right)+\left|W_{i}\right| \leq r_{S}\left(\Gamma_{H_{0}}\left(T_{i}\right) \cup X\right)+d_{G}\left(T_{i}, W_{i}\right)
\end{gathered}
$$

from which $d_{G}\left(T_{i}, W_{i}\right) \geq p_{T}\left(T_{i}\right)-r_{S}\left(X \cup \Gamma_{H_{0}}\left(T_{i}\right)\right)$. Therefore $G$ has at least $\sum_{i=1}^{q}\left[p_{T}\left(T_{i}\right)-r_{S}\left(X \cup \Gamma_{H_{0}}\left(T_{i}\right)\right)\right]$ edges connecting $T-Y$ and $S-X$, and $G$ has at least $\widetilde{m}_{S}(X)+\widetilde{m}_{T}(Y)-d_{G_{0}}(X, Y)$ edges with end-nodes in $X$ or in $Y$, from which the inequality in (2) follows.

Sufficiency. Let $\mathcal{H}_{0}:=\left\{V^{\prime} \subseteq V:\right.$ no arc of $\overrightarrow{H_{0}}$ enters $\left.V^{\prime}\right\}$. Then $\mathcal{H}_{0}$ is closed under taking union and intersection. In the following definition of set-function $p_{0}$, we have $X \subseteq S, Y \subseteq T$, and $y \in T$.

$$
p_{0}\left(V^{\prime}\right):= \begin{cases}\max \left\{p_{T}(y)-r_{S}(X), m_{T}(y)-|X|+d_{H_{0}}(y)\right\} & \text { if } V^{\prime}=X+y \in \mathcal{H}_{0}, \\ p_{T}(Y)-r_{S}(X) & \text { if } V^{\prime}=X \cup Y \in \mathcal{H}_{0},|Y| \geq 2 \\ 0 & \text { otherwise. }\end{cases}
$$

The definition of $p_{0}$ implies that $p_{0}\left(V^{\prime}\right)$ can be positive only if $V^{\prime} \in \mathcal{H}_{0}$.

Lemma 3. The set-function $p_{0}$ is positively $T$-intersecting supermodular.

Proof. Proof. Let $X_{1}, X_{2}$ be subsets of $S$ and let $Y_{1}, Y_{2}$ be subsets of $T$ for which $Y_{1} \cap Y_{2} \neq \emptyset$. Suppose that $p_{0}\left(V_{i}\right)>0$ for $V_{i}=X_{i} \cup Y_{i}(i=1,2)$. Then each of the sets $V_{1}, V_{2}, V_{1} \cap V_{2}$, and $V_{1} \cup V_{2}$ belongs to $\mathcal{H}_{0}$. We distinguish three cases.

Case $1 p_{0}\left(V_{i}\right)=p_{T}\left(Y_{i}\right)-r_{S}\left(X_{i}\right)$ for $i=1,2$. Then

$$
\begin{gathered}
p_{0}\left(V_{1}\right)+p_{0}\left(V_{2}\right)=\left[p_{T}\left(Y_{1}\right)-r_{S}\left(X_{1}\right)\right]+\left[p_{T}\left(Y_{2}\right)-r_{S}\left(X_{2}\right)\right] \leq \\
p_{T}\left(Y_{1} \cap Y_{2}\right)-r_{S}\left(X_{1} \cap X_{2}\right)+p_{T}\left(Y_{1} \cup Y_{2}\right)-r_{S}\left(X_{1} \cup X_{2}\right) \leq p_{0}\left(V_{1} \cap V_{2}\right)+p_{0}\left(V_{1} \cup V_{2}\right) .
\end{gathered}
$$


Case $2 p_{0}\left(V_{i}\right)>p_{T}\left(Y_{i}\right)-r_{S}\left(X_{i}\right)$ for $i=1,2$. Then $Y_{1}=Y_{2}=\{y\}$ for some $y \in T$, and $p_{0}\left(V_{i}\right)=$ $m_{T}(y)-\left|X_{i}\right|+d_{H_{0}}(y)$. We have

$$
\begin{gathered}
p_{0}\left(V_{1}\right)+p_{0}\left(V_{2}\right)=m_{T}(y)-\left|X_{1}\right|+d_{H_{0}}(y)+m_{T}(y)-\left|X_{2}\right|+d_{H_{0}}(y)= \\
m_{T}(y)-\left|X_{1} \cap X_{2}\right|+d_{H_{0}}(y)+m_{T}(y)-\left|X_{1} \cup X_{2}\right|+d_{H_{0}}(y) \leq p_{0}\left(V_{1} \cap V_{2}\right)+p_{0}\left(V_{1} \cup V_{2}\right) .
\end{gathered}
$$

Case $3 p_{0}\left(V_{1}\right)=p_{T}\left(Y_{1}\right)-r_{S}\left(X_{1}\right)$ and $p_{0}\left(V_{2}\right)>p_{T}\left(Y_{2}\right)-r_{S}\left(X_{2}\right)$. (The situation is analogous when the indices $i=1,2$ are interchanged.) Then $Y_{2}=\{y\}$ for some $y \in T$ and $y \in Y_{1}$. Since

$$
r_{S}\left(X_{1} \cup X_{2}\right)-r_{S}\left(X_{1}\right) \leq\left|\left(X_{1} \cup X_{2}\right)-X_{1}\right|=\left|X_{2}\right|-\left|X_{1} \cap X_{2}\right|,
$$

we have $-r_{S}\left(X_{1}\right)-\left|X_{2}\right| \leq-r_{S}\left(X_{1} \cup X_{2}\right)-\left|X_{1} \cap X_{2}\right|$ and hence

$$
\begin{gathered}
p_{0}\left(V_{1}\right)+p_{0}\left(V_{2}\right)=\left[p_{T}\left(Y_{1}\right)-r_{S}\left(X_{1}\right)\right]+\left[m_{T}(y)-\left|X_{2}\right|+d_{H_{0}}(y)\right]= \\
{\left[p_{T}\left(Y_{1} \cup Y_{2}\right)-r_{S}\left(X_{1}\right)\right]+\left[m_{T}(y)-\left|X_{2}\right|+d_{H_{0}}(y)\right] \leq} \\
p_{T}\left(Y_{1} \cup Y_{2}\right)-r_{S}\left(X_{1} \cup X_{2}\right)+m_{T}(y)-\left|X_{1} \cap X_{2}\right|+d_{H_{0}}(y) \leq p_{0}\left(V_{1} \cup V_{2}\right)+p_{0}\left(V_{1} \cap V_{2}\right),
\end{gathered}
$$

as required.

Claim 4. $m_{S}(s) \leq d_{G_{0}}(s)$ for each $s \in S$.

Proof. Proof. By applying (2) to $Y=T, X=\{s\}$, and $\mathcal{T}=\emptyset$, the claim follows.

For $s \in S$, let $V_{s}:=\left\{v \in V-s: s v \notin F_{0}\right\}$. Note that $V_{s} \in \mathcal{H}_{0}$ for $s \in S$. Let a set-function $p_{1}$ on $V$ be defined as follows.

$$
p_{1}\left(V^{\prime}\right):= \begin{cases}m_{S}(s) & \text { if } V^{\prime}=V_{s} \text { for some } s \in S \\ p_{0}\left(V^{\prime}\right) & \text { otherwise. }\end{cases}
$$

The definition of $p_{1}$ implies that $p_{1}\left(V^{\prime}\right)$ can be positive only if $V^{\prime} \in \mathcal{H}_{0}$.

Claim 5. $p_{1}\left(V_{s}\right) \geq p_{0}\left(V_{s}\right)$ holds for every $s \in S$.

Proof. Proof. Consider first the case when $V_{s} \cap T=\{y\}$ for some $y \in T$. By applying (2) to $X=S-s$, $Y=\{y\}$, and $\mathcal{T}=\emptyset$, we get

$$
m_{T}(y)-|S-s|+d_{H_{0}}(y)=m_{T}(y)-d_{G_{0}}(S-s, y) \leq m_{S}(s) .
$$

By applying (2) to $X=S-s, Y=\emptyset$, and $\mathcal{T}=\{y\}$, we get $p_{T}(y)-r_{S}(S-s) \leq m_{S}(s)$, from which

$$
m_{S}(s) \geq \max \left\{p_{T}(y)-r_{S}(S-s), m_{T}(y)-|S-s|+d_{H_{0}}(y)\right\}=p_{0}\left(V_{s}\right) .
$$

Second, assume that $\left|V_{s} \cap T\right| \geq 2$. By applying (2) to $X=S-s, Y=\emptyset$, and $\mathcal{T}=\left\{V_{s} \cap T\right\}$, we get

$$
p_{0}\left(V_{s}\right)=p_{T}\left(V_{s} \cap T\right)-r_{S}(S-s) \leq m_{S}(s) .
$$

Claim 6. The set-function $p_{1}$ is positively ST-crossing supermodular.

Proof. Proof. It follows from Claim 5 that $p_{1}$ arises from $p_{0}$ by increasing its values on sets $V_{s}(s \in S)$. Let $V^{\prime} \subset V$ be a set which is $S T$-crossing with $V_{s}$ (in particular, $V^{\prime}$ and $V_{s}$ are not comparable). Then $S \nsubseteq V_{s} \cup V^{\prime}$ and hence $V^{\prime} \cap S \subseteq V_{s} \cap S$. Therefore $V^{\prime} \cap T \nsubseteq V_{s} \cap T$, that is, there is an element $t \in\left(V^{\prime}-V_{s}\right) \cap T$. Since $s t$ is an arc of $\overrightarrow{H_{0}}$ entering $V^{\prime}$, we conclude that $p_{1}\left(V^{\prime}\right)=0$, implying that $p_{1}$ is indeed positively $S T$-crossing supermodular.

Let $\nu$ denote the maximum total $p_{1}$-value of $S T$-independent sets.

Lemma 7. $\nu=\gamma$. 
Proof. Proof. Since the family $\mathcal{L}=\left\{V_{s}: s \in S\right\}$ is $S T$-independent, $\nu \geq \widetilde{p}_{1}(\mathcal{L})=\widetilde{m}_{S}(S)=\gamma$. Suppose indirectly that $\nu>\gamma$ and let $\mathcal{I}$ be an $S T$-independent family for which $\widetilde{p}_{1}(\mathcal{I})=\nu$. We can assume that $|\mathcal{I}|$ is minimal in which case $p_{1}\left(V^{\prime}\right)>0$ for each $V^{\prime} \in \mathcal{I}$.

Claim 8. There are no two $T$-intersecting members $V_{1}$ and $V_{2}$ of $\mathcal{I}$ for which $p_{1}\left(V_{i}\right)=p_{0}\left(V_{i}\right)(i=1,2)$.

Proof. Proof. Suppose indirectly the existence of such $T$-intersecting members $V_{1}$ and $V_{2}$ of $\mathcal{I}$. Since $\mathcal{I}$ is $S T$-independent, we must have $S \subseteq V_{1} \cup V_{2}$ and hence $p_{0}\left(V_{1} \cup V_{2}\right)=0$. Since $p_{0}$ is positively $T$-intersecting supermodular,

$$
\begin{gathered}
p_{1}\left(V_{1}\right)+p_{1}\left(V_{2}\right)=p_{0}\left(V_{1}\right)+p_{0}\left(V_{2}\right) \leq \\
p_{0}\left(V_{1} \cap V_{2}\right)+p_{0}\left(V_{1} \cup V_{2}\right)=p_{0}\left(V_{1} \cap V_{2}\right) \leq p_{1}\left(V_{1} \cap V_{2}\right) .
\end{gathered}
$$

Now $\mathcal{I}^{\prime}=\mathcal{I}-\left\{V_{1}, V_{2}\right\}+\left\{V_{1} \cap V_{2}\right\}$ is also $S T$-independent and $\widetilde{p}_{1}\left(\mathcal{I}^{\prime}\right) \geq \widetilde{p}_{1}(\mathcal{I})$, but we must have equality by the optimality of $\mathcal{I}$, contradicting the minimality of $|\mathcal{I}|$.

We say that a member $V^{\prime} \in \mathcal{I}$ is of Type I if $V^{\prime}=X_{t}+t$ for some $t \in T$ and $X_{t} \subseteq S$ and

$$
p_{1}\left(X_{t}+t\right)=p_{0}\left(X_{t}+t\right)=m_{T}(t)-\left|X_{t}\right|+d_{H_{0}}(t)>p_{T}(t)-r_{S}\left(X_{t}\right) .
$$

Let $\mathcal{I}_{1}(\subseteq \mathcal{I})$ denote the family of sets of Type I. Claim 8 implies that if $X_{1}+t_{1} \in \mathcal{I}_{1}$ and $X_{2}+t_{2} \in \mathcal{I}_{1}$ for which $X_{1}+t_{1} \neq X_{2}+t_{2} \quad\left(X_{i} \subseteq S, t_{i} \in T\right)$, then $t_{1} \neq t_{2}$. Let

$$
Y:=\left\{t \in T: \text { there is a member } X_{t}+t \in \mathcal{I}_{1}\right\} .
$$

Note that $|Y|=\left|\mathcal{I}_{1}\right|$.

We say that a member $V^{\prime} \in \mathcal{I}$ is of Type II if

$$
p_{1}\left(V^{\prime}\right)=p_{0}\left(V^{\prime}\right)=p_{T}\left(V^{\prime} \cap T\right)-r_{S}\left(V^{\prime} \cap S\right) .
$$

Let $\mathcal{I}_{2}=\left\{V_{1}, V_{2}, \cdots, V_{q}\right\}(\subseteq \mathcal{I})$ denote the family of sets of Type II. Let

$$
\mathcal{T}:=\left\{T_{1}, \ldots, T_{q}\right\} \text { where } T_{i}:=V_{i} \cap T \text { for } i=1, \ldots, q .
$$

Since $p_{1}\left(V_{i}\right)>0$, the members of $\mathcal{T}$ are non-empty. Furthermore, Claim 8 implies that $\mathcal{T}$ is a subpartition of $T-Y$.

Let $\mathcal{I}_{3}:=\mathcal{I}-\left(\mathcal{I}_{1} \cup \mathcal{I}_{2}\right)$. The members of $\mathcal{I}_{3}$ are called of Type III. Then each member $V^{\prime}$ of $\mathcal{I}_{3}$ is of form $V^{\prime}=V_{s}$ for some $s \in S$ such that $m_{S}(s)=p_{1}\left(V^{\prime}\right)>p_{0}\left(V^{\prime}\right)$. Let

$$
X:=\left\{s \in S: V_{s} \in \mathcal{I}_{3}\right\} .
$$

It follows from the definitions that $\mathcal{I}_{1}, \mathcal{I}_{2}$, and $\mathcal{I}_{3}$ form a partition of $\mathcal{I}$.

Claim 9. Let $t \in Y$ and $X_{t}+t \in \mathcal{I}_{1}$. Then $X \subseteq X_{t}$.

Proof. Proof. Suppose indirectly that there is an element $s \in X-X_{t}$. By the $S T$-independence of the sets $X_{t}+t$ and $V_{s}$, the element $t$ cannot be in $V_{s}$. Therefore the arc $s t$ belongs to $\overrightarrow{F_{0}}$. Since $s t$ enters $X_{t}+t$, we have $p_{1}\left(X_{t}+t\right)=0$, a contradiction.

Claim 10. $\sum\left[\left|X_{t}\right|-d_{H_{0}}(t): t \in Y\right] \geq d_{G_{0}}(X, Y)$.

Proof. Proof. What we prove is that $\left|X_{t}\right|-d_{H_{0}}(t) \geq d_{G_{0}}(X, t)$ for $t \in Y$ and $X_{t}+t \in \mathcal{I}_{1}$. Since no arc of $\overrightarrow{H_{0}}$ enters $X_{t}+t$ and since $X \subseteq X_{t}$ by Claim 9 , we have

$$
\left|X_{t}\right|-d_{H_{0}}(t)=\left|X_{t}\right|-d_{H_{0}}\left(X_{t}, t\right)=d_{G_{0}}\left(X_{t}, t\right) \geq d_{G_{0}}(X, t),
$$

as required.

Claim 11. $X \cup \Gamma_{H_{0}}\left(T_{i}\right) \subseteq V_{i} \cap S$ holds for each $i=1, \ldots, q$. 
Proof. Proof. As $V_{i} \in \mathcal{H}_{0}$, we have $\Gamma_{H_{0}}\left(T_{i}\right) \subseteq V_{i} \cap S$. If, indirectly, there is an $s \in X-V_{i}$, then the $S T$-independence of $V_{s}$ and $V_{i}$ implies that $V_{s} \cap V_{i} \cap T=\emptyset$. In this case, an element $t \in V_{i} \cap T$ cannot be in $V_{s}$ implying that $s t \in \overrightarrow{F_{0}}$. But then $p_{1}\left(V_{i}\right)=0$, contradicting the property $p_{1}\left(V^{\prime}\right)>0$ for each $V^{\prime} \in \mathcal{I}$.

Recall that $\mathcal{T}$ is a subpartition of $T-Y$. This and the last two claims imply

$$
\begin{gathered}
\gamma<\nu=\widetilde{p}_{1}(\mathcal{I})=\widetilde{p}_{1}\left(\mathcal{I}_{1}\right)+\widetilde{p}_{1}\left(\mathcal{I}_{2}\right)+\widetilde{p}_{1}\left(\mathcal{I}_{3}\right)= \\
\sum\left[m_{T}(t)-\left|X_{t}\right|+d_{H_{0}}(t): X_{t}+t \in \mathcal{I}_{1}\right]+\sum_{i=1}^{q}\left[p_{T}\left(T_{i}\right)-r_{S}\left(V_{i} \cap S\right)\right]+\sum\left[m_{S}(s): V_{s} \in \mathcal{I}_{3}\right] \leq \\
\sum\left[m_{T}(t): X_{t}+t \in \mathcal{I}_{1}\right]-d_{G_{0}}(X, Y)+\sum_{i=1}^{q}\left[p_{T}\left(T_{i}\right)-r_{S}\left(X \cup \Gamma_{H_{0}}\left(T_{i}\right)\right)\right]+\widetilde{m}_{S}(X)= \\
\widetilde{m}_{T}(Y)-d_{G_{0}}(X, Y)+\sum_{i=1}^{q}\left[p_{T}\left(T_{i}\right)-r_{S}\left(X \cup \Gamma_{H_{0}}\left(T_{i}\right)\right)\right]+\widetilde{m}_{S}(X),
\end{gathered}
$$

in a contradiction with (2), completing the proof of the lemma.

Claim 12. If $p_{1}\left(V^{\prime}\right)$ is positive, then $\overrightarrow{G_{0}}$ covers $V^{\prime}$.

Proof. Proof. As already observed after $(4), V^{\prime} \in \mathcal{H}_{0}$. Assume to the contrary that $\overrightarrow{G_{0}}$ does not cover $V^{\prime}$. As $G_{0}$ denotes the bipartite complement of $H_{0}$, this is only possible if $V^{\prime} \cap T=\emptyset$ or $S \subseteq V^{\prime}$.

If $V^{\prime}=V_{s}$ for some $s \in S$, then $s \notin V^{\prime}$, hence $V^{\prime} \cap T=\emptyset$. This means that $s t \in F_{0}$ for each $t \in T$. By applying (2) to $X=\{s\}, Y=T$ and $\mathcal{T}=\emptyset$, we get $p_{1}\left(V^{\prime}\right)=m_{S}(s) \leq 0$, a contradiction.

Therefore, we must have $p_{1}\left(V^{\prime}\right)=p_{0}\left(V^{\prime}\right)$. As $p_{0}$ was defined to be 0 for sets not intersecting $T$, we can assume that $S \subseteq V^{\prime}$ holds. If $p_{0}\left(V^{\prime}\right)=p_{T}\left(V^{\prime} \cap T\right)-r_{S}\left(V^{\prime} \cap S\right)$, then (2), when applied to $Y=\emptyset, X=S$ and $\mathcal{T}=\left\{V^{\prime} \cap T\right\}$, gives $p_{0}\left(V^{\prime}\right)=p_{T}\left(V^{\prime} \cap T\right)-r_{S}(S) \leq 0$, a contradiction. Therefore $p_{0}\left(V^{\prime}\right)$ is defined by the first line of (3). Hence $V^{\prime}=S+y$ for some $y \in T$ and $p_{0}\left(V^{\prime}\right)=m_{T}(y)-|S|+d_{H_{0}}(y)$. Now (2), when applied to $Y=\{y\}, X=S$ and $\mathcal{T}=\emptyset$, gives $p_{0}\left(V^{\prime}\right)=m_{T}(y)-|S|+d_{H_{0}}(y)=m_{T}(y)-d_{G_{0}}(S, y) \leq 0$, thus leading to a contradiction again.

By Claim 12, Theorem 1 can be applied to $p_{1}$. This means that there is a digraph $D=(V, A)$ on $V$ with $\nu=\gamma S T$-arcs that covers $p_{1}$, that is, $\varrho_{D}\left(V^{\prime}\right) \geq p_{1}\left(V^{\prime}\right)$ for every subset $V^{\prime} \subseteq V$. Let $G=(S, T ; E)$ denote the undirected bipartite graph underlying $D$.

Claim 13. $d_{G}(s)=m_{S}(s)$ for every $s \in S$ and $d_{G}(t)=m_{T}(t)$ for every $t \in T$.

Proof. Proof. Since $d_{G}(s)=\delta_{D}(s) \geq \varrho_{D}\left(V_{s}\right) \geq p_{1}\left(V_{s}\right)=m_{S}(s)$ for every $s \in S$, we have $\widetilde{m}_{S}(S)=|E|=$ $\sum\left[d_{G}(s): s \in S\right] \geq \widetilde{m}_{S}(S)$, from which $d_{G}(s)=m_{S}(s)$ follows for every $s \in S$.

Since $d_{G}(t)=\varrho_{D}(t) \geq \varrho_{D}\left(\Gamma_{H_{0}}(t)+t\right) \geq p_{0}\left(\Gamma_{H_{0}}(t)+t\right) \geq m_{T}(t)$ for every $t \in T$, we have $\widetilde{m}_{T}(T)=$ $|E|=\sum\left[d_{G}(t): t \in T\right] \geq \widetilde{m}_{T}(T)$, from which $d_{G}(t)=m_{T}(t)$ follows for every $t \in T$.

Claim 14. The bigraph $G^{+}=\left(S, T ; E+F_{0}\right)$ is simple.

Proof. Proof. The minimality of $D$ implies that each $\operatorname{arc}$ of $D$ enters a subset $V^{\prime}$ with $p_{1}\left(V^{\prime}\right)>0$. Since $p_{1}\left(V^{\prime}\right)$ can be positive only if no arc of $\overrightarrow{H_{0}}$ enters $V^{\prime}$, we can conclude that no edge of $G$ is parallel to an edge of $H_{0}$.

Suppose indirectly that there are two parallel edges $e$ and $e^{\prime}$ of $G$ connecting $s$ and $t$ for some $s \in S$ and $t \in T$. Let $X:=\Gamma_{H_{0}}(t)$. Then $p_{1}(X+t) \geq m_{T}(t)=\varrho_{D}(t)$. For $V^{\prime}=X+s+t$, we have $\varrho_{D}(t)-2 \geq \varrho_{D}\left(V^{\prime}\right) \geq p_{1}\left(V^{\prime}\right) \geq p_{1}(X+t)-1 \geq m_{T}(t)-1=\varrho_{D}(t)-1$, a contradiction.

Claim 15. $r_{S}\left(\Gamma_{G^{+}}(Y)\right) \geq p_{T}(Y)$ for every subset $Y \subseteq T$.

Proof. Proof. Let $X:=\Gamma_{G^{+}}(Y)$ and $V^{\prime}:=Y \cup X$. Then $0=\varrho_{D}\left(V^{\prime}\right) \geq p_{1}\left(V^{\prime}\right) \geq p_{T}(Y)-r_{S}(X)$, from which the claim follows.

We conclude that $G$ meets all the requirements of the theorem, and the proof is complete. 


\subsection{Variations}

\subsubsection{Degree-specification only on $S$}

With the proof technique used above, one can derive the following variation where the degrees are specified only for the nodes in $S$. Namely, the definition of $p_{0}$ in (3) should be modified as follows.

$$
p_{0}\left(V^{\prime}\right):= \begin{cases}p_{T}(Y)-r_{S}(X) & \text { if } V^{\prime}=X \cup Y \in \mathcal{H}_{0}, \quad X \subseteq S, Y \subseteq T \\ 0 & \text { otherwise. }\end{cases}
$$

Theorem 16. We are given a simple bigraph $H_{0}=\left(S, T ; F_{0}\right)$, a matroid $M_{S}=\left(S, r_{S}\right)$, a positively intersecting supermodular function $p_{T}$ on $T$, and a degree-specification $m_{S}$ on $S$ for which $\widetilde{m}_{S}(S)=\gamma$. There is a bigraph $G=(S, T ; E)$ fitting $m_{S}$ for which $G^{+}=G+H_{0}$ is simple and $M_{S}$-covers $p_{T}$ if and only if

$$
m_{S}(s)+d_{H_{0}}(s) \leq|T| \text { for every } s \in S
$$

and

$$
\begin{gathered}
\tilde{m}_{S}(X)+\sum_{i=1}^{q}\left[p_{T}\left(T_{i}\right)-r_{S}\left(X \cup \Gamma_{H_{0}}\left(T_{i}\right)\right)\right] \leq \gamma \\
\text { whenever } X \subseteq S \text { and } \mathcal{T}=\left\{T_{1}, \ldots, T_{q}\right\} \text { a subpartition of } T .
\end{gathered}
$$

One reason why we do not go into the details is that the proof is quite similar to (and, in fact, slightly simpler than) the proof of Theorem 2. Another reason is that, in a forthcoming work [3], we solve a common generalization of Theorems 2 and 16 where, instead of degree-specifications, there are both upper and lower bounds for the degrees of all nodes in $S \cup T$.

\subsubsection{Fully supermodular $p_{T}$}

In the special case when $p_{T} \equiv 0$, it suffices to require the inequality in $(2)$ only for the empty $\mathcal{T}$, in which case Theorem 2 reduces to the following classic result (which actually holds for non-simple bigraphs, too).

Theorem 17 (Ore [12]). A simple bigraph $G_{0}=\left(S, T ; E_{0}\right)$ has a subgraph fitting a degree-specification $\left(m_{S}, m_{T}\right)$ with $\widetilde{m}_{S}(S)=\widetilde{m}_{T}(T)=\gamma$ if and only if

$$
\widetilde{m}_{S}(X)+\widetilde{m}_{T}(Y)-d_{G_{0}}(X, Y) \leq \gamma \text { whenever } X \subseteq S, Y \subseteq T .
$$

The content of the next result is that the condition in Theorem 2 can also be simplified when $p_{T}$ is fully supermodular.

Theorem 18. We are given a simple bigraph $H_{0}=\left(S, T ; F_{0}\right)$, a matroid $M_{S}=\left(S, r_{S}\right)$, a fully supermodular function $p_{T}$ on $T$, and a degree-specification $m_{V}=\left(m_{S}, m_{T}\right)$ for which $\widetilde{m}_{S}(S)=\widetilde{m}_{T}(T)=\gamma$. There is a bigraph $G=(S, T ; E)$ fitting $m_{V}$ for which $G^{+}=G+H_{0}$ is simple and $M_{S}$-covers $p_{T}$ if and only if (8) holds and

$$
\begin{gathered}
\widetilde{m}_{S}(X)+\widetilde{m}_{T}(Y)-d_{G_{0}}(X, Y)+p_{T}\left(T_{0}\right)-r_{S}\left(X \cup \Gamma_{H_{0}}\left(T_{0}\right)\right) \leq \gamma \\
\text { whenever } Y \subseteq T, X \subseteq S, T_{0} \subseteq T-Y,
\end{gathered}
$$

where $G_{0}$ is the bipartite complement of $H_{0}$.

Proof. Proof. Conditions (8) and (9) correspond to the special cases of Condition (2) when $|\mathcal{T}|=0$ and $|\mathcal{T}|=1$, respectively. Therefore their necessity was proved earlier. To see sufficiency, by Theorem 2 it suffices to show that (2) holds in general. Suppose, indirectly, that there are $X, Y$, and $\mathcal{T}$ violating (2). Assume that $|\mathcal{T}|$ is minimal. Then (8) and (9) imply that $|\mathcal{T}| \geq 2$. Let $T_{1}, T_{2}$ be two members of $\mathcal{T}$. Since

$$
p_{T}\left(T_{1} \cup T_{2}\right)-r_{S}\left(X \cup \Gamma_{H_{0}}\left(T_{1} \cup T_{2}\right)\right) \geq p_{T}\left(T_{1}\right)+p_{T}\left(T_{2}\right)-r_{S}\left(X \cup \Gamma_{H_{0}}\left(T_{1}\right)\right)-r_{S}\left(X \cup \Gamma_{H_{0}}\left(T_{2}\right)\right),
$$

the unchanged sets $X, Y$ and the partition $\mathcal{T}^{\prime}$ obtained from $\mathcal{T}$ by replacing $T_{1}$ and $T_{2}$ with the single set $T_{1} \cup T_{2}$ also violate (2), contradicting the minimal choice of $\mathcal{T}$.

It is worth formulating Theorem 18 in the special case when $H_{0}$ has no edges. 
Corollary 19. We are given a matroid $M_{S}=\left(S, r_{S}\right)$, a fully supermodular function $p_{T}$ on $T$, and a degreespecification $m_{V}=\left(m_{S}, m_{T}\right)$ for which $\widetilde{m}_{S}(S)=\widetilde{m}_{T}(T)=\gamma$. There is a simple bigraph $G=(S, T ; E)$ fitting $m_{V}$ and $M_{S}$-covering $p_{T}$ if and only if

$$
\widetilde{m}_{S}(X)+\widetilde{m}_{T}(Y)-|X||Y| \leq \gamma \text { whenever } X \subseteq S, Y \subseteq T
$$

and

$$
\widetilde{m}_{S}(X)+\widetilde{m}_{T}(Y)-|X||Y|+p_{T}\left(T_{0}\right)-r_{S}(X) \leq \gamma \text { whenever } Y \subseteq T, X \subseteq S, T_{0} \subseteq T-Y .
$$

If, in addition, $p_{T}$ is monotone non-decreasing, then $T_{0}$ in (11) can be chosen to be $T_{0}=T-Y$, that is,

$$
\widetilde{m}_{S}(X)+\widetilde{m}_{T}(Y)-|X||Y|+p_{T}(T-Y)-r_{S}(X) \leq \gamma \text { whenever } X \subseteq S, Y \subseteq T .
$$

Proof. Proof. The first part is a special case of Theorem 18. When $p_{T}$, in addition, is monotone nondecreasing in the second part, we can choose $T_{0}$ in (11) as large as possible, that is, $T_{0}=T-Y$.

\section{Matroidal max term rank}

Let $\mathcal{G}\left(m_{S}, m_{T}\right)$ denote the set of simple bigraphs $G=(S, T ; E)$ fitting a degree-specification $\left(m_{S}, m_{T}\right)$ with $\widetilde{m}_{S}(S)=\widetilde{m}_{T}(T)=\gamma$. It follows from Theorem 17 that $\mathcal{G}\left(m_{S}, m_{T}\right)$ is non-empty if and only if (10) holds. In [1] (Theorem 26), we reformulated Ryser's classic max term rank formula in graph theoretic language.

Theorem 20 (Ryser). Let $\ell \leq|T|$ be an integer. Suppose that $\mathcal{G}\left(m_{S}, m_{T}\right)$ is non-empty. Then $\mathcal{G}\left(m_{S}, m_{T}\right)$ has a member $G$ with matching number $\nu(G) \geq \ell$ if and only if

$$
\widetilde{m}_{S}(X)+\widetilde{m}_{T}(Y)-|X||Y|+(\ell-|X|-|Y|) \leq \gamma \text { whenever } X \subseteq S, Y \subseteq T .
$$

Moreover, (13) holds if the inequality in it is required only when $X$ consists of the $i$ largest values of $m_{S}$ and $Y$ consists of the $j$ largest values of $m_{T}(i=0,1, \ldots,|S|, j=0,1, \ldots,|T|)$.

We keep using graph terminology, but the original expression (max term rank) of Ryser is retained. Our present goal is to extend Ryser's theorem in two directions. In the augmentation version an initial bigraph is to be augmented while in the matroidal form the matching is expected to cover a basis of a matroid $M_{S}$ on $S$ and a basis of matroid $M_{T}$ on $T$. Actually, we shall integrate the two generalizations into one single framework.

In what follows, $M_{S}=\left(S, r_{S}\right)$ and $M_{T}=\left(T, r_{T}\right)$ will be matroids of rank $\ell$. In [1], the complementary set-function $p$ of a set-function $b$ was defined by $p(Y):=b(S)-b(S-Y)$. Clearly, $b$ is submodular if and only if $p$ is supermodular, and $p$ is monotone non-decreasing if and only if $b$ is monotone non-decreasing. The complementary function $p_{T}$ of the rank function $r_{T}$ of $M_{T}$ is called the co-rank function of $M_{T}$. It can easily be shown that $p_{T}(Y)=\min \left\{|Y \cap B|: B\right.$ a basis of $\left.M_{T}\right\}$.

The following extension of Edmonds' matroid intersection theorem [6] will be used. For notational convenience, the bipartite graph in the theorem is denoted by $G^{+}$.

Theorem 21 (Brualdi, [4]). Let $G^{+}=\left(S, T ; E^{+}\right)$be a bigraph with a matroid $M_{S}=\left(S, r_{S}\right)$ on $S$ and with a matroid $M_{T}=\left(T, r_{T}\right)$ on $T$ for which $r_{S}(S)=r_{T}(T)=\ell$. There is a matching of $G^{+}$covering bases of $M_{S}$ and $M_{T}$ if and only if

$$
\begin{gathered}
r_{S}\left(X^{\prime}\right)+r_{T}\left(Y^{\prime}\right) \geq \ell \\
\text { whenever } X^{\prime} \cup Y^{\prime} \text { hits every edge of } G^{+}\left(X^{\prime} \subseteq S, Y^{\prime} \subseteq T\right) .
\end{gathered}
$$

We need the following equivalent version of Theorem 21.

Lemma 22. Let $G^{+}=\left(S, T ; E^{+}\right)$be a bigraph. Let $M_{S}$ be a matroid on $S$ with rank function $r_{S}$ and $M_{T}$ a matroid on $T$ with co-rank function $p_{T}$ for which $r_{S}(S)=p_{T}(T)=\ell$. There is a matching of $G^{+}$ covering bases of $M_{S}$ and $M_{T}$ if and only if

$$
r_{S}\left(\Gamma_{G^{+}}(Y)\right) \geq p_{T}(Y) \text { for every } Y \subseteq T .
$$


Proof. Proof. The necessity is straightforward. The sufficiency follows from Theorem 21 once we show that (14) holds. Since $X^{\prime} \cup Y^{\prime}$ hits every edge of $G^{+}$, for $Y:=T-Y^{\prime}$ we have $\Gamma_{G^{+}}(Y) \subseteq X^{\prime}$. Therefore (15) implies that $r_{S}\left(X^{\prime}\right) \geq r_{S}\left(\Gamma_{G^{+}}(Y)\right) \geq p_{T}(Y)=r_{T}(T)-r_{T}\left(Y^{\prime}\right)=\ell-r_{T}\left(Y^{\prime}\right)$ and hence (14) indeed holds.

Theorem 23. We are given a simple bigraph $H_{0}=\left(S, T ; F_{0}\right)$, a matroid $M_{S}=\left(S, r_{S}\right)$ and a matroid $M_{T}=\left(T, r_{T}\right)$ with $r_{S}(S)=r_{T}(T)=\ell$, and a degree-specification $m_{V}=\left(m_{S}, m_{T}\right)$ on $V:=S \cup T$ for which $\widetilde{m}_{S}(S)=\widetilde{m}_{T}(T)=\gamma$. There is a bigraph $G=(S, T ; E)$ fitting $m_{V}$ for which $G^{+}=G+H_{0}$ is simple and includes a matching covering a basis of $M_{S}$ and a basis of $M_{T}$ if and only if (8) holds and

$$
\begin{gathered}
\tilde{m}_{S}(X)+\widetilde{m}_{T}(Y)-d_{G_{0}}(X, Y)+\ell-r_{S}\left(X^{\prime}\right)-r_{T}\left(Y^{\prime}\right) \leq \gamma \\
\text { whenever } X \subseteq X^{\prime} \subseteq S, Y \subseteq Y^{\prime} \subseteq T \text {, and } X^{\prime} \cup Y^{\prime} \text { hits all the edges of } H_{0},
\end{gathered}
$$

where $G_{0}$ is the bipartite complement of $H_{0}$.

Proof. Proof. Necessity. Suppose that the requested bigraph $G$ and its $\ell$-element matching $M$ exist. The number of edges of $G$ with at least one end-node in $X \cup Y$ is at least $\widetilde{m}_{S}(X)+\widetilde{m}_{T}(Y)-d_{G_{0}}(X, Y)$. The number of edges in $M$ with at least one end-node in $X^{\prime} \cup Y^{\prime}$ is at most $r_{S}\left(X^{\prime}\right)+r_{T}\left(Y^{\prime}\right)$. Therefore $M$ has at least $\ell-r_{S}\left(X^{\prime}\right)-r_{T}\left(Y^{\prime}\right)$ elements connecting $S-X^{\prime}$ and $T-Y^{\prime}$. But these elements must be in $E$ since $X^{\prime} \cup Y^{\prime}$ hits all edges of $H_{0}$. Therefore the total number of edges of $G$ is at least $\widetilde{m}_{S}(X)+\widetilde{m}_{T}(Y)-$ $d_{G_{0}}(X, Y)+\ell-r_{S}\left(X^{\prime}\right)-r_{T}\left(Y^{\prime}\right)$, and (16) follows.

Sufficiency. Let $p_{T}$ denote the co-rank function of $M_{T}$, that is, $p_{T}(Z)=\ell-r_{T}(T-Z)$ for $Z \subseteq T$. Note that $p_{T}$ is fully supermodular.

Claim 24. Condition (9) is satisfied.

Proof. Proof. For the present $p_{T}$, Condition (9) requires

$$
\widetilde{m}_{S}(X)+\widetilde{m}_{T}(Y)-d_{G_{0}}(X, Y)+\ell-r_{T}\left(T-T_{0}\right)-r_{S}\left(X \cup \Gamma_{H_{0}}\left(T_{0}\right)\right) \leq \gamma
$$

No sets $X \subseteq S, Y \subseteq T$, and $T_{0} \subseteq T-Y$ can violate this inequality since then, by letting $Y^{\prime}:=T-T_{0}$ and $X^{\prime}:=X \cup \Gamma_{H_{0}}\left(T_{0}\right)$, the quadruple $\left(X, Y, X^{\prime}, Y^{\prime}\right)$ would violate $(16)$.

By Theorem 18, there is a bigraph $G$ fitting $m_{V}$ for which $G^{+}=G+H_{0}$ is simple and $M_{S}$-covers $p_{T}$. The latter property, by definition, means that (15) holds, and therefore Lemma 22 implies that $G^{+}$has a requested matching.

When $m_{V} \equiv 0$ and $\gamma=0$, it suffices to require (16) only for $X=Y=\emptyset$ in which case it transforms to

$$
\ell-r_{S}\left(X^{\prime}\right)-r_{T}\left(Y^{\prime}\right) \leq 0 \text { whenever } X^{\prime} \subseteq S, Y^{\prime} \subseteq T \text {, and } X^{\prime} \cup Y^{\prime} \text { hits all the edges of } H_{0},
$$

which is the same as (14). In other words, Theorem 23 may be considered as a straight generalization of Brualdi's theorem.

The content of the next corollary is that in the special case of Theorem 8 when $F_{0}=\emptyset$ it suffices to require (16) only in a simplified form.

Corollary 25. Let $S$ and $T$ be two disjoint sets and $\left(m_{S}, m_{T}\right)$ a degree-specification on $S \cup T$ for which $\widetilde{m}_{S}(S)=\widetilde{m}_{T}(T)=\gamma$ and $\mathcal{G}\left(m_{S}, m_{T}\right)$ is non-empty, that is, (10) holds. Let $M_{S}=\left(S, r_{S}\right)$ and $M_{T}=\left(T, r_{T}\right)$ be matroids for which $r_{S}(S)=r_{T}(T)=\ell$. There is a simple bigraph $G=(S, T ; E)$ fitting $\left(m_{S}, m_{T}\right)$ that includes a matching covering bases of $M_{S}$ and $M_{T}$ if and only if

$$
\widetilde{m}_{S}(X)+\widetilde{m}_{T}(Y)-|X||Y|+\ell-r_{S}(X)-r_{T}(Y) \leq \gamma
$$

holds for every $X \subseteq S$ and $Y \subseteq T$. 
Proof. Proof. Consider Theorem 23 in the special case when $F_{0}=\emptyset$. Then the bipartite complement $G_{0}$ of $H_{0}$ is a complete bigraph and hence $d_{G_{0}}(X, Y)=|X||Y|$. Therefore Condition (10) requested in the corollary is the same as Condition (8) requested in Theorem 23. Furthermore (19) is the special case of (16) when $X^{\prime}=X$ and $Y^{\prime}=Y$, and hence (19) is necessary.

We claim, conversely, that (19) implies (16). Indeed, if $X \subseteq X^{\prime}$ and $Y \subseteq Y^{\prime}$ violate the inequality in (16), then the monotonicity of matroid rank functions imply that $\gamma<\widetilde{m}_{S}(X)+\widetilde{m}_{T}(Y)-|X||Y|+\ell-$ $r_{S}\left(X^{\prime}\right)-r_{T}\left(Y^{\prime}\right) \leq \widetilde{m}_{S}(X)+\widetilde{m}_{T}(Y)-|X||Y|+\ell-r_{S}(X)-r_{T}(Y)$, contradicting (19). Therefore the requested bigraph exists by Theorem 23.

Note that the inequalities in Conditions (10) and (19) can be integrated into the following single form:

$$
\widetilde{m}_{S}(X)+\widetilde{m}_{T}(Y)-|X||Y|+\left(\ell-r_{S}(X)-r_{T}(Y)\right)^{+} \leq \gamma .
$$

By specializing Theorem 23 to the case when $M_{S}$ and $M_{T}$ are $\ell$-uniform matroids on $S$ and $T$, respectively, one obtains the following.

Corollary 26. We are given a simple bigraph $H_{0}=\left(S, T ; F_{0}\right)$, an integer $\ell$, and a degree-specification $\left(m_{S}, m_{T}\right)$ for which $\widetilde{m}_{S}(S)=\widetilde{m}_{T}(T)=\gamma$. There is a bigraph $G=(S, T ; E)$ fitting $\left(m_{S}, m_{T}\right)$ for which $G^{+}=G+H_{0}$ is simple and includes an $\ell$-element matching if and only if (8) holds and

$$
\begin{gathered}
\widetilde{m}_{S}(X)+\widetilde{m}_{T}(Y)-d_{G_{0}}(X, Y)+\ell-\left|X^{\prime}\right|-\left|Y^{\prime}\right| \leq \gamma \\
\text { whenever } X \subseteq X^{\prime} \subseteq S, Y \subseteq Y^{\prime} \subseteq T \text {, and } X^{\prime} \cup Y^{\prime} \text { hits all the edges of } H_{0},
\end{gathered}
$$

where $G_{0}$ is the bipartite complement of $H_{0}$.

Proof. Proof. Consider Theorem 23 in the special case when $M_{S}$ and $M_{T}$ are $\ell$-uniform matroids on $S$ and on $T$, respectively. Since matroid rank functions are subcardinal, (21) is implied by (16) and hence (21) is necessary.

We claim, conversely, that (21) implies (16), that is, $\alpha+\ell-r_{S}\left(X^{\prime}\right)-r_{T}\left(Y^{\prime}\right) \leq \gamma$ where $\alpha:=\widetilde{m}_{S}(X)+$ $\widetilde{m}_{T}(Y)-d_{G_{0}}(X, Y)$. Indeed, if $\max \left\{\left|X^{\prime}\right|,\left|Y^{\prime}\right|\right\} \leq \ell$, then $\alpha+\ell-r_{S}\left(X^{\prime}\right)-r_{T}\left(Y^{\prime}\right)=\alpha+\ell-\min \left\{\ell,\left|X^{\prime}\right|\right\}-$ $\min \left\{\ell,\left|Y^{\prime}\right|\right\}=\alpha+\ell-\left|X^{\prime}\right|-\left|Y^{\prime}\right| \leq \gamma$, where the last inequality follows by (21). If $\max \left\{\left|X^{\prime}\right|,\left|Y^{\prime}\right|\right\}>\ell$, and, say, $\left|X^{\prime}\right|>\ell$, then $\alpha+\ell-r_{S}\left(X^{\prime}\right)-r_{T}\left(Y^{\prime}\right)=\alpha+\ell-\min \left\{\ell,\left|X^{\prime}\right|\right\}-\min \left\{\ell,\left|Y^{\prime}\right|\right\} \leq \alpha+\ell-\ell-0=\alpha \leq \gamma$, where the last inequality follows by $(8)$. Therefore the requested bigraph exists by Theorem 23 .

Acknowledgements We are grateful to Richard Brualdi for the valuable information on the topic. Special thanks are due to Zoltán Szigeti for carefully checking the details of a first draft. The two anonymous referees provided several useful comments. We gratefully acknowledge their great contribution.

The research was supported by the Hungarian Scientific Research Fund - OTKA, No K109240. The work of the first author was financed by a postdoctoral fellowship provided by the Hungarian Academy of Sciences.

\section{References}

[1] K. Bérczi and A. Frank, Supermodularity in unweighted graph optimization I: Branchings and matchings. Mathematics of Operations Research, this issue.

[2] K. Bérczi and A. Frank, Supermodularity in unweighted graph optimization III: Highly-connected digraphs. Mathematics of Operations Research, this issue.

[3] K. Bérczi and A. Frank, Supermodularity in unweighted graph optimization IV: Algorithms (2017), in preparation.

[4] R.A. Brualdi, Admissible mappings between dependence spaces, Proceedings of the London Mathematical Society, Vol 3, No. 2 (1970) 296-312.

[5] R.A. Brualdi and J.A. Ross, On Ryser's Maximum Term Rank Formula, Linear Algebra and its Applications, Vol. 29 (1980) 33-38. 
[6] J. Edmonds, Submodular functions, matroids, and certain polyhedra, in: Combinatorial Structures and their Applications (R. Guy, H. Hanani, N. Sauer, and J. Schönheim, eds.), Gordon and Breach, New York (1970) 69-87.

[7] L.R. Ford and D.R. Fulkerson, Flows in Networks, Princeton Univ. Press, Princeton NJ., 1962.

[8] A. Frank, Connections in Combinatorial Optimization, Oxford University Press, 2011 (ISBN 978-019-920527-1). Oxford Lecture Series in Mathematics and its Applications, 38.

[9] A. Frank and T. Jordán, Minimal edge-coverings of pairs of sets, Journal of Combinatorial Theory, Ser. B, Vol. 65, No. 1 (1995, September) 73-110.

[10] D. Gale, A theorem on flows in networks, Pacific Journal of Mathematics, Vol. 10 (1957) 1073-1082.

[11] R.R. Kamalian and V.V. Mkrtchyan, On complexity of special maximum matchings constructing, Discrete Mathematics, Vol. 308, No. 10 (2008) 1792-1800.

[12] O. Ore, Studies on directed graphs, I, Annals of Mathematics, Vol. 63 (1956) 383-406.

[13] D. Pálvölgyi, Partitioning to three matchings of given size is NP-complete for bipartite graphs, Acta Universitatis Sapientiae, Informatica, Vol. 6, No. 2 (2014) 206-209.

[14] G.J. Puleo, Complexity of a disjoint matching problem on bipartite graphs, Information Processing Letters, Vol. 116, No. 10 (October 2016) 649-652.

[15] H.J. Ryser, Combinatorial properties of matrices of zeros and ones, Canadian Journal of Mathematics, Vol. 9 (1957) 371-377.

[16] H.J. Ryser, The term rank of a matrix, Canadian Journal of Mathematics, Vol. 10 (1958) 57-65.

[17] L.A. Végh and A.A. Benczúr, Primal-dual approach for directed vertex connectivity augmentation and generalizations, ACM Transactions on Algorithms, Vol. 4, No. 2 (2008) 1-21. 